Ab-initio modeling of

water-semiconductor interfaces for direct solar-to-chemical energy conversion

B. Wood, T. Ogitsu, E. Schwegler

July 26, 2010

SPIE Optics + Photonics

San Diego, CA, United States

August 1, 2010 through August 5, 2010 
This document was prepared as an account of work sponsored by an agency of the United States government. Neither the United States government nor Lawrence Livermore National Security, LLC, nor any of their employees makes any warranty, expressed or implied, or assumes any legal liability or responsibility for the accuracy, completeness, or usefulness of any information, apparatus, product, or process disclosed, or represents that its use would not infringe privately owned rights. Reference herein to any specific commercial product, process, or service by trade name, trademark, manufacturer, or otherwise does not necessarily constitute or imply its endorsement, recommendation, or favoring by the United States government or Lawrence Livermore National Security, LLC. The views and opinions of authors expressed herein do not necessarily state or reflect those of the United States government or Lawrence Livermore National Security, LLC, and shall not be used for advertising or product endorsement purposes. 


\title{
Ab-initio modeling of water-semiconductor interfaces for direct solar-to-chemical energy conversion
}

\author{
Brandon C. Wood, Tadashi Ogitsu, and Eric Schwegler \\ Quantum Simulations Group \\ Lawrence Livermore National Laboratory, Livermore, CA 94550
}

\begin{abstract}
We perform extensive density-functional theory total-energy calculations and ab-initio molecular dynamics simulations to evaluate the stability and reactivity of surface oxides and hydroxides of $\operatorname{InP}(001)$ for photoelectrochemical water cleavage. In order to achieve maximal accuracy, our simulations include the full interface between the semiconductor surface and liquid water. Certain oxide contaminants are found to have a dramatic impact on the surface reactivity, pointing to the importance of surface oxide and hydroxide intermediates in facilitating the water-dissociation component of the hydrogen evolution process. Our results are used to relate the chemical activity of the surface towards water dissociation to the oxygen bond topology. The importance of the liquid hydrogen-bond network near the interface is discussed, particularly in relation to the generation of local configurations favorable for dissociative water adsorption on $\operatorname{InP}(001)$.
\end{abstract}

Keywords: Photoelectrochemical, hydrogen production, III-V semiconductors, indium phosphide

\section{INTRODUCTION}

Development of efficient, environmentally responsible processes for the production of chemical fuel using sunlight has been identified by the U.S. Department of Energy as a strategic goal for a secure and sustainable energy future. ${ }^{1}$ Semiconductor-based photoelectrochemical (PEC) hydrogen production from water is particularly attractive, thanks to a relatively simple device design and a wealth of data available for engineering efficient photon harvesting. ${ }^{2-4}$ Nevertheless, practical implementations of PEC devices have been limited, in part because the hydrogen evolution processes at the electrode-water interface are poorly understood.

A schematic illustration of a semiconductor-based PEC cell is given in Fig. 1. The semiconductor serves a dual function in the device as a photon harvester and catalytic surface for hydrogen evolution from water. ${ }^{1,3}$ An effective PEC photocatalyst must meet certain initial requirements for high efficiency. First, the band gap must be optimized with respect to the solar spectrum to maximize collection. Second, valence and conduction band edges should closely match the redox potentials of water, which may be modulated by electrolytes or surface states. Recognizing this, past efforts have concentrated on engineering the band gap and level alignment to the redox potential of water. Nevertheless, although results have demonstrated that one can achieve sufficiently high solar-to-fuel conversion efficiency, ${ }^{5}$ this efficiency has come at the expense of short device lifetime due to fast degradation of the electrode. ${ }^{6-8}$

It has long been suggested that oxygen, which is present in high concentrations as a contaminant in (001) surfaces of III-V semiconductors, may play a significant role in motivating both the surface photocorrosion and the catalytic water splitting reaction. ${ }^{7,9}$ Given the apparent connection between high catalytic activity and high potential for surface degradation in III-V systems, as well as the barrier it poses to device adoption, evaluating this potential role of oxygen represents a high priority for developing a complete understanding of PEC device shortcomings under real-world operating conditions. Nevertheless, the role of surface oxygen has not been explored in detail theoretically.

Here we use a combination of ab-initio molecular dynamics simulations and total-energy calculations based on density functional theory (DFT) to understand the microscopic hydrogen evolution reaction mechanism at a model electrode/electrolyte interface consisting of oxidized $\operatorname{InP}(001)$ and water. The choice of $\operatorname{InP}(001)$ was

Send correspondence to B.C.W. (wood37@llnl.gov) 


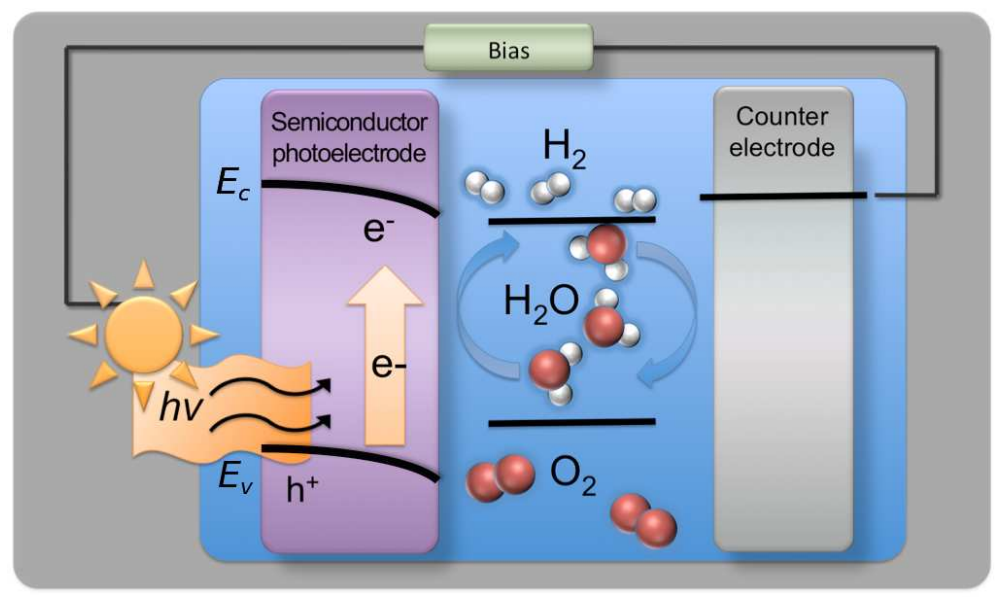

Figure 1. Schematic of a simple PEC device. Photoexcitation occurs in the semiconductor photoelectrode, which is immersed with a counter-electrode in an open-circuit configuration in a liquid water electrolyte. The valence and conduction band edges, which sandwich the redox potentials of water, bend near the interface to facilitate electron-hole separation. Catalytic evolution of hydrogen occurs at the semiconductor-water interface.

motivated by its known water-splitting activity (although its band gap of $1.35 \mathrm{eV}$ is slightly too small for use in a practical PEC device). Our results are used to explore the effect of oxide and hydroxide surface morphologies on surface stability and reactivity, and to evaluate the role of interfacial water and surface dynamics in their local realization.

\section{METHODS}

Results are based on density functional theory within the plane-wave pseudopotential formalism, as calculated using the Quantum-ESPRESSO code. ${ }^{10}$ The Perdew-Burke-Ernzerhof (PBE) exchange-correlation functional was used. ${ }^{11}$ Ultrasoft pseudopotentials ${ }^{12}$ were used for all elements, and semi-core $d$ states were included in the electronic valence description for indium. For the ionic relaxation calculations, forces were converged to within $10^{-4} \mathrm{Ry} / a_{0}$, and $12 \AA$ of vacuum separation was inserted between periodic slab images.

Molecular dynamics results were obtained within the Car-Parrinello framework, ${ }^{13}$ using $\mathrm{D}_{2} \mathrm{O}$ in lieu of $\mathrm{H}_{2} \mathrm{O}$ to permit larger time steps. The fictitious electronic mass was chosen as 700 a.u., using a time step of 12 a.u. All simulations were run within the canonical $N V T$ ensemble, with temperatures maintained using Nose-Hoover chains. ${ }^{14}$ Simulations were run at $400 \mathrm{~K}$ in order to properly reproduce the structural properties of liquid water ${ }^{15}$ (boiling temperature is elevated in molecular dynamics simulations). Since the system is semiconducting, a weak thermostat was also added to the electronic degrees of freedom in order to maintain separation from the ionic degrees of freedom over long simulation trajectories.

For simulations of the full semiconductor-water surface, seven semiconductor layers were included in a surface slab configuration, with 16 atoms per layer (112 atoms total for the semiconductor, not including the surface oxide/hydroxide atoms). Both exposed surfaces were indium-rich $\operatorname{InP}(001)$. In-plane axes were aligned along the [110] and [111] crystallographic directions. A total of 142 water molecules were inserted between periodic slab images, filling an intermediate region about $16.5 \AA$ wide (the density was chosen to match the experimental density of liquid water). To generate the initial configuration for the interface, we first ran classical simulations for bulk water using the TIP4P potential. The water molecules were then inserted into the full model structure, and the system was equilibrated for $1 \mathrm{ps}$ with the surface degrees of freedom kept frozen. Another 3 ps of equilibration was then performed with all degrees of freedom active before the production run. Simulations were run for 15 ps each. 


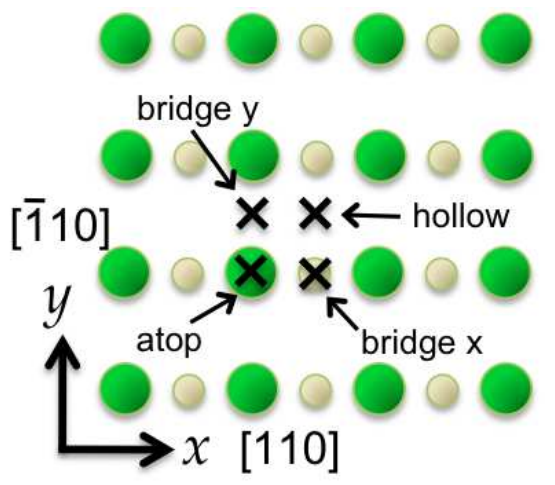

Figure 2. High-symmetry sites for initial adsorption on unreconstructed $\operatorname{InP}(001)$. Indium atoms (large, dark green) form the surface layer, with phosphorous atoms (small, light gold) in the subsurface layer.

Table 1. Energies of relaxed oxide structures (eV per oxygen) relative to the reference state (see text for details). Symmetry site for initial oxygen placement is also listed, along with the final location of the oxygen with respect to the top indium layer (surface or subsurface) and the oxide bond topology (In-O-In bridging versus In-O-P bridging). Compare to Fig. 3.

\begin{tabular}{cccccc}
\hline $\begin{array}{c}\text { Initial adsorption } \\
\text { site }\end{array}$ & $\begin{array}{c}\text { Energy (eV per O) } \\
\mathrm{O}_{2} \text { reference }\end{array}$ & $\begin{array}{c}\text { Energy (ev per O) } \\
\mathrm{H}_{2} / \mathrm{H}_{2} \mathrm{O} \text { reference }\end{array}$ & Location & \% In-O-In & \% In-O-P \\
\hline Atop(1) & -2.465 & +0.894 & both & 50 & 50 \\
Atop(2) & -2.512 & +0.848 & surface & 75 & 25 \\
Atop(3) & -2.558 & +0.801 & surface & 75 & 25 \\
Bridge x & -3.018 & +0.341 & subsurface & 0 & 100 \\
Bridge y & -1.969 & +1.390 & surface & 100 & 0 \\
Hollow(1) & -2.554 & +0.806 & subsurface & 0 & 100 \\
Hollow(2) & -2.704 & +0.655 & subsurface & 0 & 100 \\
\hline
\end{tabular}

\section{OXIDES OF INDIUM PHOSPHIDE (001)}

In order to extract low-energy oxide configurations, we adsorbed atomic oxygen on high-symmetry sites of an initially unreconstructed In-rich $\operatorname{InP}(001)$ surface slab. The atoms were then allowed to relax to their localminimum configuration. Atop, hollow, and both bridge sites were tested in the high coverage (1.0 ML) limit, using five-layer $\mathrm{InP}(001)$ slabs with four atoms per layer and four total adsorbate atoms (see Fig. 2 for schematic of initial adsorption sites). The odd number of layers was to ensure both exposed surfaces were In-rich, thereby minimizing spurious interactions between periodic slab images. Site symmetry was broken by displacing the four oxygen atoms off their ideal sites in symmetric and antisymmetric patterned configurations. Doing so exposed additional metastable structures for the atop and hollow sites, giving seven total morphologies.

The energies and atomic configurations of the relaxed oxide structures are given in Table 1 and Fig. 3 . Energies are given with respect to two reference configurations. The first is with respect to $\operatorname{InP}(001) \delta(2 \times 4)-$ the most commonly observed In-rich reconstruction in vacuum ${ }^{16,17}$ - and molecular $\mathrm{O}_{2}$. The second is with respect to $\operatorname{InP}(001) \delta(2 \times 4), \mathrm{H}_{2} \mathrm{O}$, and $\mathrm{H}_{2}$. All tested configurations show that incorporation of oxygen is strongly thermodynamically favored with respect to the clean surface in the presence of gaseous $\mathrm{O}_{2}$, in agreement with experimental observations of high surface oxygen contamination. ${ }^{7,9,18}$ When taken with respect to water, however, incorporation is endothermic.

Several of the tested configurations (hollow, bridge $\mathrm{x}$, and one of the atop sites) result in the oxygen in the subsurface layer rather than the surface layer (see Fig. 3). These generally feature oxygen simultaneously bonded to one indium and one phosphorous. In some of the structures (bridge $\mathrm{x}$ in particular), the oxygen pushes the indium to which it is bonded far out of the indium plane. Surface oxygens, on the other hand, generally bridge two indium atoms. The atop(1) configuration is unique in that it features half surface oxygen in an In-O-In 


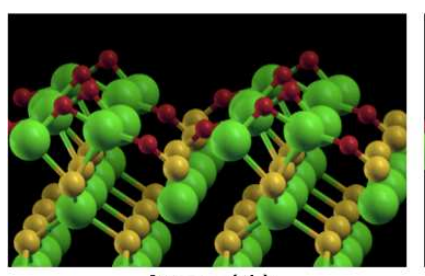

Atop(1)

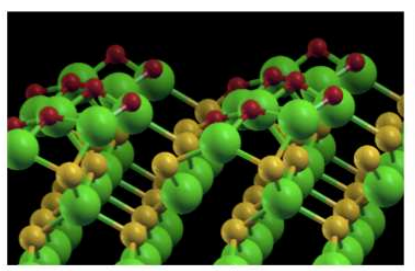

Atop(2)

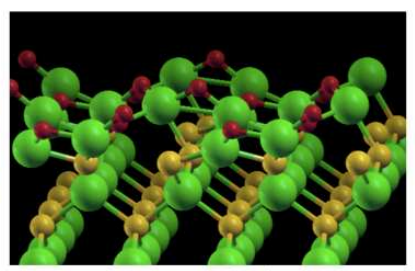

Atop(3)

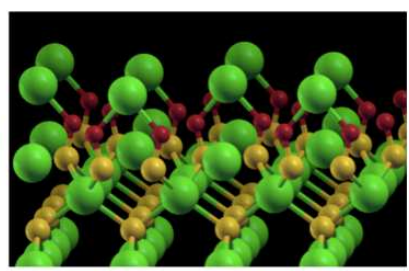

Bridge $x$

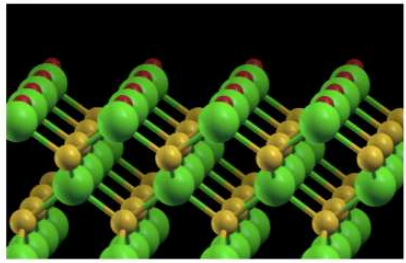

Bridge y

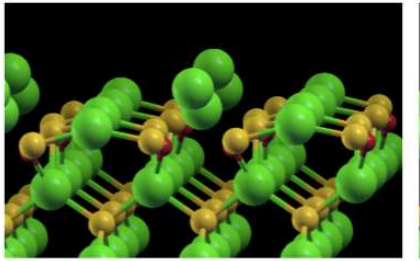

Hollow(1)

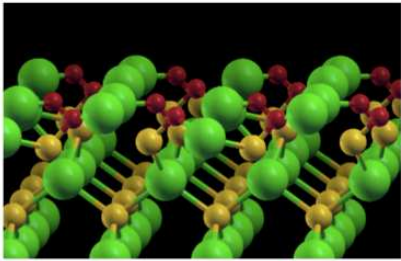

Hollow(2)

Figure 3. Relaxed structures for the oxides listed in Table 1. Views are in the $x z$ plane. Color scheme: $\mathrm{In}=$ green, $\mathrm{P}=$ gold, $\mathrm{O}=$ red.

topology and half subsurface oxygen in an In-O-P topology. Table 1 also indicates that oxygen is generally more stable in the subsurface, most likely reflecting the strength of the In-O-P bond with respect to the In-O-In bond. Consistent with this interpretation, the least energetically favorable configuration (bridge y) features all In-O-In bonds, whereas the most favorable (bridge $\mathrm{x}$ ) features all $\mathrm{In}-\mathrm{O}-\mathrm{P}$ bonds.

To test whether oxide formation affects reactivity for water dissociation, single water molecules were adsorbed near the oxygen sites on each of the morphologies in Table 1. In each case, oxygen bond topology proves an excellent predictor of gas-phase dissociative adsorption of water: oxygen sites in In-O-P bonds bind water weakly and do not encourage dissociation, whereas sites in In-O-In bonds promote spontaneous dissociative adsorption. For morphologies containing both types of oxide bonds, reactivity depends on the specific adsorption site. To illustrate this point, the results of water adsorption on three representative oxide surfaces-bridge x, bridge $\mathrm{y}$, atop(1) - are shown in Fig. 4. For the reactive In-O-In topologies, adsorption of water results in cleavage of one of the $\mathrm{O}-\mathrm{H}$ bonds. The cleaved hydrogen attaches to one of the surface $\mathrm{In}-\mathrm{O}-\mathrm{In}$ bridging oxygens, whereas the remaining $\mathrm{OH}$ moiety attaches to a nearby In atom (Fig. 4b\&c).

\section{HYDROXIDES OF INDIUM PHOSPHIDE (001)}

Given that the result of water reaction with certain bridge oxide structures is local surface hydroxylation, there is interest in determining the properties of fully hydroxylated surfaces. Accordingly, we repeated the above procedure, placing $\mathrm{OH}$ in high-symmetry sites to obtain the relaxed local-minimum structure. In contrast to the rich array of oxides in Table 1, only three unique relaxed configurations were found for OH coverage (bridge y, hollow, and atop). These are shown in Fig. 5. The corresponding energies are listed in Table 2, calculated with respect to two references: first, $\operatorname{InP}(001) \delta(2 \times 4)$, molecular $\mathrm{O}_{2}$, and molecular $\mathrm{H}_{2}$; and second, first, $\mathrm{InP}(001)$ $\delta(2 \times 4), \mathrm{H}_{2} \mathrm{O}$, and molecular $\mathrm{H}_{2}$. Note the similarity between the hollow and atop configurations, with the only difference being weak In-O bond formation along the $\hat{x}$ direction for some of the OH groups. This is also reflected in the near-degeneracy of the two energies.

Unlike the oxide case, hydroxide formation is thermodynamically favored both with respect to the molecular $\mathrm{H}_{2} / \mathrm{O}_{2}$ reference and respect to the $\mathrm{H}_{2} \mathrm{O} / \mathrm{H}_{2}$ reference. This means hydroxylation should be common in solution as well as vacuum. It also reflects the thermodynamic driving force for the dissociation of water on the reactive oxide surfaces, since this results in surface hydroxylation. In addition, the most stable configuration is the bridge $\mathrm{y}$, which is also the least stable oxide configuration that we tested.

The spread of the energies in Table 2 is much smaller than in Table 1 . The three structures are very similar, each featuring bridge y motifs with variations on the bonding of the remaining $\mathrm{OH}$ groups. The atop structure mixes one row of bridge $y$ with a row of atop, which features strongly interacting hydrogen bonds between the 

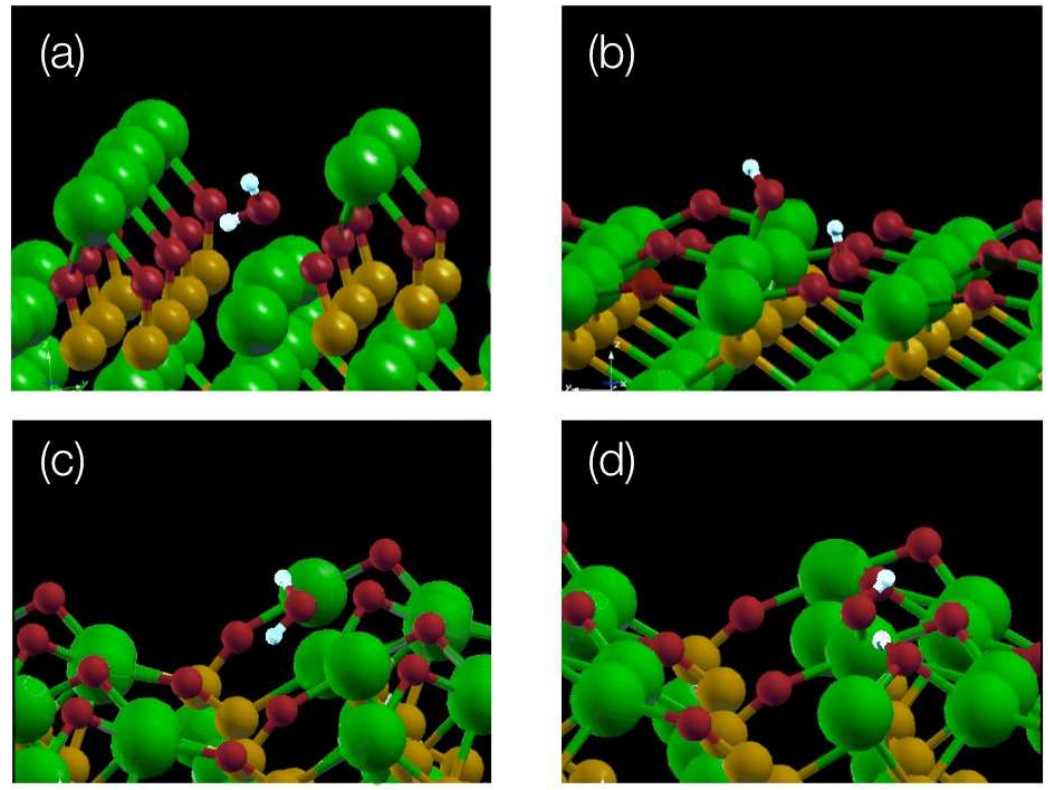

Figure 4. Relaxed structure of a single gas-phase water molecule adsorbed on three different $\operatorname{InP}(001)$ surface oxide structures. For the bridge $\mathrm{x}$ oxide (a), which features exclusively In-O-P bond topologies, the water molecule remains intact. For the bridge y oxide (b), which features exclusively In-O-In bond topologies, water dissociation occurs, resulting in local surface hydroxylation. For the atop(1) oxide (c) \& (d), which features half In-O-In and half In-O-P topologies, water dissociation does not occur if adsorption happens near the In-O-P bond (c), but does occur if adsorption happens near the In-O-In bond (d). Color scheme: $\mathrm{In}=$ green, $\mathrm{P}=$ gold, $\mathrm{O}=$ red, $\mathrm{H}=$ white.

Table 2. Energies of relaxed hydroxide structures (eV per $\mathrm{OH}$ ) relative to the reference state (see text for details). Compare to Fig. 5 .

\begin{tabular}{ccc}
\hline \hline $\begin{array}{c}\text { Initial adsorption } \\
\text { site }\end{array}$ & $\begin{array}{c}\text { Energy }(\mathrm{eV} \text { per } \mathrm{OH}) \\
\mathrm{H}_{2} / \mathrm{O}_{2} \text { reference }\end{array}$ & $\begin{array}{c}\text { Energy }(\mathrm{eV} \text { per } \mathrm{OH}) \\
\mathrm{H}_{2} / \mathrm{H}_{2} \mathrm{O} \text { reference }\end{array}$ \\
\hline Atop & -3.630 & -0.271 \\
Bridge $y$ & -3.692 & -0.333 \\
Hollow & -3.629 & -0.270 \\
\hline
\end{tabular}

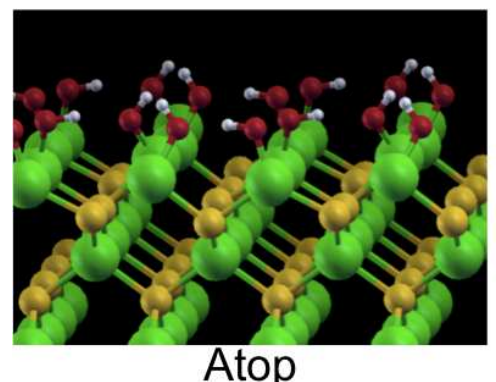

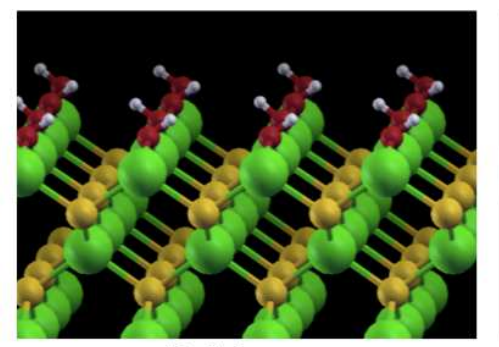

Bridge y

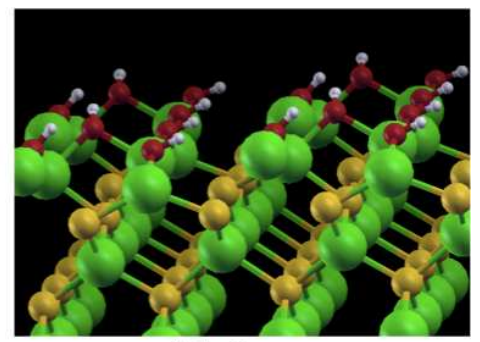

Hollow

Figure 5. Relaxed structures for the hydroxides listed in Table 2. Views are in the $x z$ plane. Color scheme: In=green, $\mathrm{P}=$ gold, $\mathrm{O}=$ red, $\mathrm{H}=$ white. 
dangling $\mathrm{OH}$ groups. The hollow structure mixes one row of bridge y with a row of half-coverage bridge y and a half-coverage bridge $\mathrm{x}$. Since these differ only in if and where the oxygens form a second $\mathrm{In}-\mathrm{O}$ bond for certain of the $\mathrm{OH}$ groups, this bond is necessarily very weak. The low barrier to breaking and forming a second In-O bond for an $\mathrm{OH}$ group translates to a shallow free energy surface, where multiple morphologies may coexist and freely exchange among themselves.

\section{MOLECULAR DYNAMICS}

Since the photocatalytic reactions take place at the interface with a finite-temperature liquid, the surface morphology and electronic structure are necessarily complex and highly dynamic. This casts significant doubt on the reliability of zero-temperature gas-phase adsorption studies. Accordingly, we supplemented our zero-temperature results with calculations derived from ab-initio molecular dynamics.

Having extracted oxide and hydroxide configurations of interest, we proceeded to run ab-initio molecular dynamics calculations of the full semiconductor-water interface to examine the influence of dynamics on the reactivity and stability of the surface. In order to isolate the two oxide bond topologies and examine them individually, we ran two oxide surface simulations, one using the low-energy bridge $\mathrm{x}$ configuration (100\% In$\mathrm{O}-\mathrm{P})$ and the other using the higher-energy bridge y configuration $(100 \% \mathrm{In}-\mathrm{O}-\mathrm{In})$. For the hydroxide surface, we ran only one simulation (bridge y), recognizing the fact that the shallow free energy surface should mean realization of the other morphologies in the course of the dynamics.

\subsection{Oxide surface}

As expected, the In-O-In surface oxide demonstrated much higher reactivity than the In-O-P subsurface oxide, with dissociation events 1-2 orders of magnitude more frequent. In fact, statistics of cleavage events were sufficient to gather information about the likely reaction sites. Figure 6 maps out these sites in detail. The periodicity of the underlying lattice is clearly visible in the in-plane directions. It is also clear that the reaction takes place near the interface, as expected. Upon further examination, three interfacial peaks are visible in the direction perpendicular to the surface plane. These correspond to the first three layers formed in the liquid water. The peak closest to the actual interface covers the bulk of the cleavage events, but some of the events also take place in the second liquid layer, with a tiny fraction also in the third. The existence of non-negligible water splitting activity as far as $4-5 \AA$ from the actual surface reflects of the medium-range order of liquid water and suggests a dynamical correlation with molecules closer to the interface. However, the Figure represents a time average of all cleavage events, making it impossible to discern the specific nature of this correlation.

The mechanism of dissociative water adsorption on the reactive In-O-In oxide is depicted in Fig. 7. The reaction begins with the adsorption of a water molecule on a surface indium site to form a bond between the water oxygen (O1) and the indium. The water molecule is initially oriented such that one of the O1-H bonds lies generally parallel to the surface, with the other pointing upwards into the solution. The parallel-lying O1-H bond is thereby free to engage in hydrogen bonding with one of the surface oxygens (O2). The subsequent cleavage occurs via proton transfer across the $\mathrm{O} 1-\mathrm{H} \cdots \mathrm{O} 2$ complex, such that the covalent and hydrogen bonds are exchanged. The result is the hydroxylation of two neighboring indium atoms, the first of which (the one to which the water $\mathrm{O} 1$ was originally bound) forms an atop hydroxide, and the second of which forms either an atop or bridge y hydroxide. Since the atop and bridge y are nearly isoenergetic, this choice is determined by the local structure visited during the dynamics and appears to be strongly dependent on number and strength of the hydrogen bonds formed with neighboring water molecules.

For many water cleavage events, the reaction culminates in two surface hydroxyl groups $(\mathrm{O} 1-\mathrm{H}$ and $\mathrm{O} 2-\mathrm{H})$. However, there are also instances in which $\mathrm{O} 2$ further binds to a second-layer phosphorous and is pulled into the subsurface. This phenomenon can be seen in Fig. 7e \& $\mathrm{f}$. We can also track it by quantifying the number of In-O-P bridge configurations in the reactive oxide sample, since the existence of these owes exclusively to oxygens being pulled into the subsurface.

In summary, there is a competition between three possibilities for the product hydroxide groups: first, formation of a bridge y hydroxide configuration; second, formation of an atop hydroxide which strongly hydrogenbonds with the solution; and third, a subsurface hydroxide that bridges In and P. Qualitatively, the choice between 


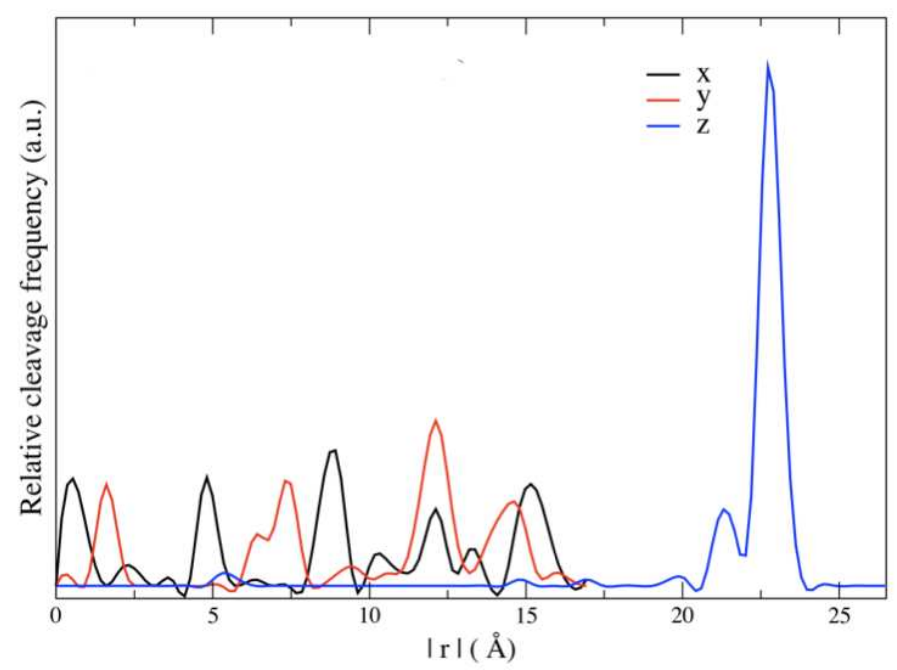

Figure 6. Profile of frequency of $\mathrm{O}-\mathrm{H}$ bond cleavage in water versus location, as projected along the three Cartesian axes ( $x$ and $y$ correspond to the [110] and [110] crystal axes, respectively). Data is for $\operatorname{InP}(001)$ with bridge y surface oxide. The surface layer begins at $z=25 \AA$.
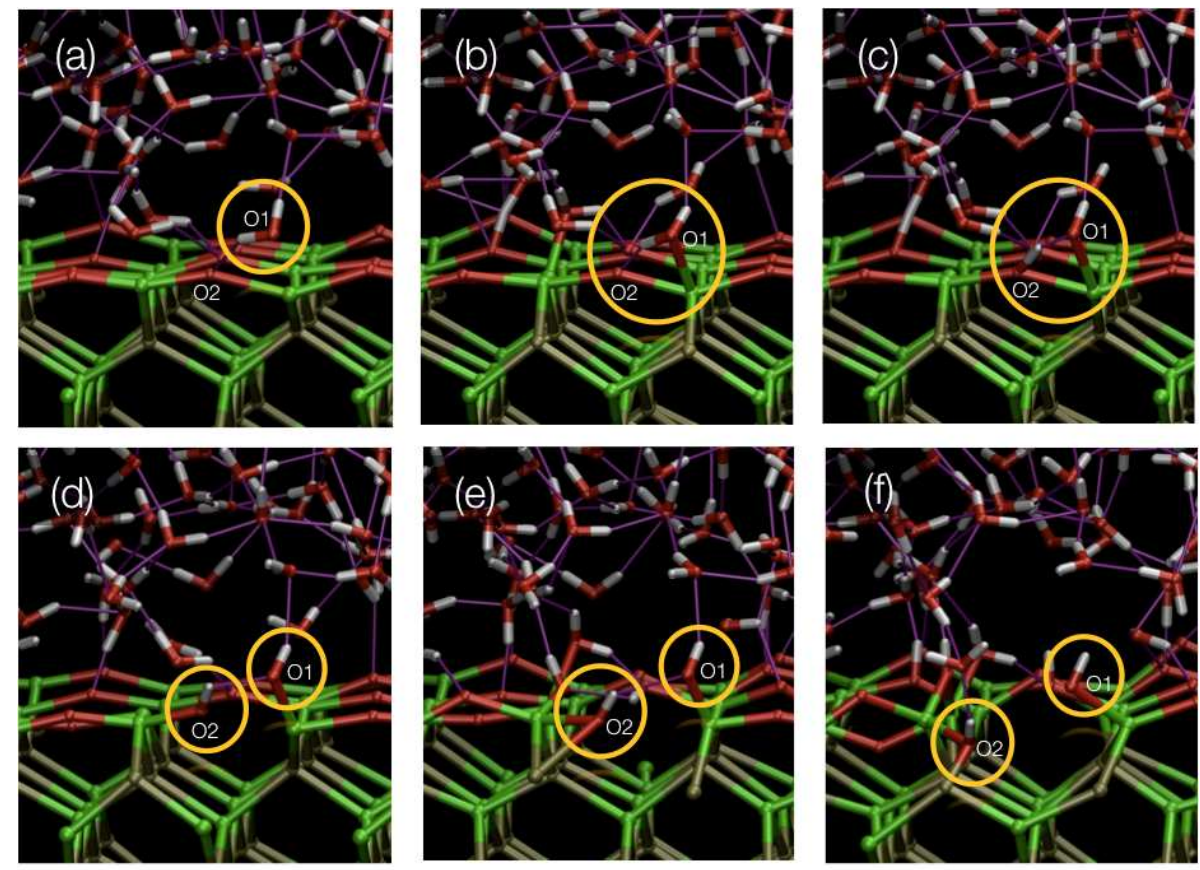

Figure 7. Mechanism of water dissociation on a bridge y oxide of $\operatorname{InP}(001)$. O1 and $\mathrm{O} 2$ refer to the oxygen atoms originally associated with the water molecule and the oxide surface, respectively. (a) \& (b) O1 binds to indium, and a hydrogen bond is formed with O2. (c) The water molecule transfers a hydrogen across the O1-H. .O2 complex. (d) The In-O2 bond breaks. (e) \& (f) Optionally, O2 is pulled below the surface to form a bond with P. The color scheme follows Fig. 4. 
these three seems to be related to the nature of the surrounding hydrogen-bond network. To be pulled into the subsurface, the product oxygen must not be engaged in significant hydrogen bonding. On the other hand, to be converted into the atop hydroxyl configuration, a product oxygen must be engaged in very strong hydrogen bonding. For intermediate cases, the in-plane bridge y configuration is preferred (although the preexistence of a bridge y structure precludes formation of a second, pushing the system towards one of the other two possibilities). Since we have already established that subsurface oxygen that bridges In and P is not generally reactive, the choice the system eventually makes can have significant consequences on further surface catalytic activity.

\subsection{Hydroxide surface}

Although we start the simulation with all hydroxide groups in the bridge y configuration, we quickly see conversion of several of these to the atop configuration, consistent with the zero-temperature predictions of facile exchange between the two. This exchange is bidirectional, indicating a dynamic equilibrium between the two configurations. As with the oxide simulations, whether a conversion takes place seems to largely depend on the structure of the hydrogen-bond network surrounding the hydroxide group. If the group is involved in strong hydrogen bonding, the atop configuration is preferred, whereas the converse is true for the bridge y configuration. It is likely that in addition to determining the relative stability of the two hydroxide bonding sites, hydrogen bonding lowers the reaction barrier for switching between the two by stabilizing intermediate transition states. As a result, interconversion is even more frequent than expected from the zero-temperature calculations.

In the dynamics simulation, water adsorption occurs preferentially on indium atoms that are bound to a single oxygen rather than to two oxygens. This undercoordination of indium atoms is connected to conversion of a hydroxide from a bridge y to an atop configuration, which in turn is responsible for the breaking of an In-O bond. This conversion to atop $\mathrm{OH}$ is ultimately facilitated by strong hydrogen-bond formation, which stabilizes the dangling $\mathrm{OH}$ groups relative to the interface with vacuum.

Occasional dissociative adsorption of water is observed in the hydroxide simulations, although it is generally associated with temporary breaking of an $\mathrm{O}-\mathrm{H}$ surface bond to form bridge y In-O-In. Water cleavage then proceeds via an essentially identical mechanism to that observed in the reactive $\mathrm{In}-\mathrm{O}-\mathrm{In}$ oxide. Since these local-oxide configurations are relatively rare, the rate of water dissociation is significantly slower than for the reactive oxide (almost an order of magnitude). In the simulation, initial formation of the oxide from the hydroxide occurs when water in the solution dissociates away from the surface, then proceeds towards the interface where it attracts the hydrogen from the $\mathrm{O}-\mathrm{H}$ surface hydroxide to reform water. In effect, this self-buffering behavior protects against increases in local $\mathrm{OH}$ concentrations that must inevitably occur at the cathode surface as a byproduct of $\mathrm{H}_{2}$ reforming.

In addition, hydrogen atoms can sometimes transfer from a water molecule in solution across a hydrogen bond to $\mathrm{OH}$ groups on the surface. This turns surface $\mathrm{OH}$ into an indium-adsorbed $\mathrm{H}_{2} \mathrm{O}$, which may then be released back into the solution to free up an indium for further reactions. This also leaves one $\mathrm{OH}$ in the solution for further surface reactions, such as the hydroxide-to-oxide conversion discussed above. The suitability of this reaction depends on temporary stabilization via local fluctuations in the hydrogen-bond network.

\section{SUMMARY AND CONCLUSIONS}

In summary, we have performed extensive total-energy calculations and ab-initio molecular dynamics simulations of water interactions with oxide- and hydroxide-decorated surfaces of the model semiconductor $\operatorname{InP}(001)$. Whereas several unique surface oxide structures are identified, only three hydroxide configurations are found. Energy calculations suggest atomic oxygen prefers to burrow into the subsurface layer and bridge indium to phosphorous. However, a somewhat less favorable configuration involves keeping the oxygen at the surface and using it to bridge two indium atoms. The first configuration is found to be inactive towards dissociation of water molecules, whereas the second catalyzes spontaneous dissociation of water at the interface. Additional oxide configurations can be characterized in terms of these two prototypical cases, with dissociative adsorption of water occurring near sites with In-O-In bond topologies and not occurring near sites with In-O-P topologies. Dynamics on the hydroxide surfaces demonstrate facile interconversion between an In-OH-In bridge configuration and an In- $\mathrm{OH}$ atop configuration. Existence of the atop configuration is found to be instrumental for enabling binding of interfacial water, since it under-coordinates a nearby indium atom. We also discuss the role 
of the local topology of hydrogen bonds between the surface and the solution in stabilizing local transition states for water splitting. Since the specific hydrogen-bond network topology near the surface fluctuates thermally, our simulations underscore the importance of finite-temperature dynamics in gaining a complete understanding of reactions at the interface. Taken together, our results suggest that oxygen contaminants play an active role in stabilizing transition states and driving dissociative adsorption of water in contact with III-V semiconductor surfaces.

\section{ACKNOWLEDGMENTS}

The authors gratefully acknowledge helpful discussions with J. Turner, T. Deutsch, Y. Yan, and K. Kim (NREL). This work was performed under the auspices of the U.S. Department of Energy by Lawrence Livermore National Laboratory under Contract DE-AC52-07NA27344.

\section{REFERENCES}

[1] U.S. Department of Energy, "New science for a secure and sustainable energy future," DOE BESAC Report (2008).

[2] Fujishima, A. and Honda, K., "Electrochemical photolysis of water at a semiconductor electrode," $N a-$ ture 238, 37 (1972).

[3] Lewis, N. S. and Nocera, D. G., "Powering the planet: Chemical challenges in solar energy utilization," PNAS 103, 15729 (2006).

[4] Turner, J. et al., "Renewable hydrogen production," Int. J. Energy Res. 32, 379 (2008).

[5] Khaselev, O. and Turner, J. A., "A monolithic photovoltaic-photoelectrochemical device for hydrogen production via water splitting," Science 280, 425 (1998).

[6] Menezes, S., Miller, B., and Bachmann, K. J., "Electrodissolution and passivation phenomena in III-V semiconducting compounds," J. Vac. Sci. Technol. B 1, 48 (1983).

[7] Vigneron, J., Herlem, M., Khoumri, E. M., and Etcheberry, A., "Cathodic decomposition of InP studied by XPS," Appl. Surf. Sci. 201, 51 (2002).

[8] Lewerenz, H. J. and Schulte, K. H., "Combined photoelectrochemical conditioning and surface analysis of InP photocathodes: II. Photoelectron spectroscopy," Electrochem. Acta 47, 2639 (2002).

[9] Heller, A., "Hydrogen-evolving solar cells," Science 223, 1141 (1984).

[10] Giannozzi, P. et al., "QUANTUM ESPRESSO: A modular and open-source software project for quantum simulations of materials," J. Phys. Condens. Matt. 21, 395502 (2009).

[11] Perdew, J. P., Burke, K., and Ernzerhof, M., "Generalized gradient approximation made simple," Phys. Rev. Lett. 77, 3865 (1996).

[12] Vanderbilt, D., "Soft self-consistent pseudopotentials in a generalized eigenvalue formalism," Phys. Rev. B 41, 7892 (1990).

[13] Car, R. and Parrinello, M., "Unified approach for molecular dynamics and density-functional theory," Phys. Rev. Lett. 55, 2471 (1985).

[14] Martyna, G. J., Klein, M. L., and Tuckerman, M. E., "Nose-Hoover chains: The canonical ensemble via continuous dynamics," J. Chem. Phys. 97, 2635 (1992).

[15] Grossman, J. C., Schwegler, E., Draeger, E. W., Gygi, F., and Galli, G., "Towards an assessment of the accuracy of density functional theory for first principles simulations of water," J. Chem. Phys. 120, 300 (2004).

[16] Li, L., Fu, Q., Li, C. H., Han, B.-K., and Hicks, R. F., "Determination of $\operatorname{InP}(001)$ surface reconstructions by STM and infrared spectroscopy of adsorbed hydrogen," Phys. Rev. B 61, 10223 (2000).

[17] Chen, G., Visbeck, S. B., Law, D. C., and Hicks, R. F., "Structure-sensitive oxidation of the indium phosphide (001) surface," J. Appl. Phys. 91, 9362 (2002).

[18] Deutsch, T. G., Koval, C. A., and Turner, J. A., "III-V nitride epilayers for photoelectrochemical water splitting: GaPN and GaAsPN," J. Phys. Chem. B 110, 25297 (2006). 